\title{
X-ray absorption near edge structure spectroscopic study of Hayabusa category 3 carbonaceous particles
}

\author{
Hikaru Yabuta ${ }^{*}$, Masayuki Uesugi $^{2}$, Hiroshi Naraoka ${ }^{3}$, Motoo $_{\text {Ito }}^{4}$, A L David Kilcoyne ${ }^{5}$, Scott A Sandford ${ }^{6}$, \\ Fumio Kitajima ${ }^{3}$, Hajime Mita ${ }^{7}$, Yoshinori Takano ${ }^{8}$, Toru Yada ${ }^{2}$, Yuzuru Karouji ${ }^{2}$, Yukihiro Ishibashi ${ }^{2}$ \\ Tatsuaki Okada ${ }^{2}$ and Masanao Abe $^{2}$
}

\begin{abstract}
Analyses with a scanning transmission $x$-ray microscope (STXM) using $x$-ray absorption near edge structure (XANES) spectroscopy were applied for the molecular characterization of two kinds of carbonaceous particles of unknown origin, termed category 3, which were collected from the Hayabusa spacecraft sample catcher. Carbon-XANES spectra of the category 3 particles displayed typical spectral patterns of heterogeneous organic macromolecules; peaks corresponding to aromatic/olefinic carbon, heterocyclic nitrogen and/or nitrile, and carboxyl carbon were all detected. Nitrogen-XANES spectra of the particles showed the presence of $\mathrm{N}$-functional groups such as imine, nitrile, aromatic nitrogen, amide, pyrrole, and amine. An oxygen-XANES spectrum of one of the particles showed a ketone group. Differences in carbon- and nitrogen-XANES spectra of the category 3 particles before and after transmission electron microscopic (TEM) observations were observed, which demonstrates that the carbonaceous materials are electron beam sensitive. Calcium-XANES spectroscopy and elemental contrast mapping identified a calcium carbonate grain from one of the category 3 particles. No fluorine-containing molecular species were detected in fluorine-XANES spectra of the particles. The organic macromolecular features of the category 3 particles were distinct from commercial and/or biological 'fresh (non-degraded)' polymers, but the category 3 molecular features could possibly reflect degradation of contaminant polymer materials or polymer materials used on the Hayabusa spacecraft. However, an extraterrestrial origin for these materials cannot currently be ruled out.
\end{abstract}

Keywords: Hayabusa; Category 3 carbonaceous particles; STXM; XANES; Organic macromolecule

\section{Findings}

Introduction

A preliminary examination of asteroid Itokawa particles collected by the Hayabusa spacecraft has successfully unveiled the mineralogical, petrographic, chemical, and isotopic relationships between an S-type asteroid and ordinary LL chondrites and provided the first direct evidence that meteorites originate from asteroids (Ebihara et al. 2011; Nakamura et al. 2011; Noguchi et al. 2011; Tsuchiyama et al. 2011; Yurimoto et al. 2011). The noble gas isotopic compositions (Nagao et al. 2011) and the asteroid particle sizes and shapes (Tsuchiyama et al. 2011) have also

\footnotetext{
* Correspondence: hyabuta@ess.sci.osaka-u.ac.jp

'Department of Earth and Space Science, Osaka University, 1-1

Machikaneyama, Toyonaka 560-0043 Osaka, Japan

Full list of author information is available at the end of the article
}

recorded asteroid surface processes such as irradiation and meteoroid impacts, which are not observed in meteorites. Organic analyses of several Itokawa particles have been carried out using micro-Raman spectroscopy, Fourier transform infrared (FTIR) spectroscopy, time-of-flight secondary ion mass spectrometry (ToF-SIMS), and two-dimensional high performance liquid chromatography (2D-HPLC) (Kitajima et al. 2011; Naraoka et al. 2012). However, to date, indigenous organic compounds have not been identified from the samples, and the presence or absence of organic compounds on the asteroid Itokawa is not clear.

In addition to the asteroid particles investigated in the preliminary examination that have been classified as 'categories 1 and 2' at the Planetary Material Sample Curation Facility of the Japan Aerospace Exploration Agency (PMSCF/JAXA), 58 carbonaceous particles of unknown

\section{实}

(c) 2014 Yabuta et al.; licensee Springer. This is an Open Access article distributed under the terms of the Creative Commons Attribution License (http://creativecommons.org/licenses/by/4.0), which permits unrestricted use, distribution, and reproduction in any medium, provided the original work is properly credited. 
origin have been collected from the Hayabusa spacecraft sample catcher and classified as 'category 3' (Uesugi et al. 2014; Yada et al. 2014). In the study reported here, two category 3 particles were analyzed by a synchrotronbased scanning transmission $\mathrm{x}$-ray microscope (STXM) using $\mathrm{x}$-ray absorption near edge structure (XANES) spectroscopy. The STXM enables the quantification of chemical compositions of the submicron-sized samples as well as elemental/molecular mapping with high spatial resolution $(<30 \mathrm{~nm})$ (Kilcoyne et al. 2003). This analytical technique was originally developed for polymer materials science (e.g.; Ade et al. 1992; Urquhart et al. 1999), but it has been applied to a broad range of research areas. In cosmochemistry, STXM has been applied for organic chemical analyses of interplanetary dust particles (IDPs) (Flynn et al. 2003, 2013; Busemann et al. 2009), particles from Comet Wild 2 (Sanford et al. 2006; Cody et al. 2008a; De Gregorio et al. 2010), acid-insoluble organic solids from chondritic meteorites (Cody et al. 2008b, Cody et al. 2011), and an Antarctic ultracarbonaceous micrometeorite (Yabuta et al. 2013). Thus, an abundant database of XANES spectra for both terrestrial and extraterrestrial organic compounds is available for the chemical characterization of unknown carbonaceous samples. We present C-, N-, O-, F-, and Ca-XANES data for two category 3 particles in order to identify their molecular compositions and determine whether the materials are terrestrial or extraterrestrial in origin.

\section{Sample preparation}

The details of category 3 sample assignments are summarized by Uesugi et al. (2014). In this study, two carbonaceous particles of the category 3 samples, RA-QD02-0120 (hereafter simply 'RA') and RB-QD04-0047-02 (hereafter simply ' $R B$ '), were investigated. The samples were also analyzed by a field emission-scanning electron microscope (FE-SEM) energy dispersion spectroscopy (EDS) (Yada et al. 2014), micro-Raman and micro-FTIR spectroscopy (Kitajima et al. 2014), NanoSIMS (secondary ion mass spectrometry) (Ito et al. 2014), and transmission electron microscope (TEM) observations (Uesugi et al. 2014). The RB sample was also analyzed by ToF-SIMS (Naraoka et al. 2014). Ultra-thin sections of RA and RB with 100-nm thickness were extracted by a focused ion beam (FIB, Hitachi FB2200, PMSCF/JAXA, Chiyoda-ku, Japan) for STXM and TEM analyses (Uesugi et al. 2014). Two different sections of RA, one section before TEM (hereafter RAbeforeTEM) and another section after TEM (hereafter RA- afterTEM), were analyzed with a STXM to evaluate electron beam damage to the sample.

\section{Analytical methods}

The XANES spectra of FIB sections of RA-beforeTEM, RA-afterTEM, and RB were acquired using a STXM at beamline (BL) 5.3.2.2 at the Advanced Light Source (ALS), Lawrence Berkeley National Laboratory, USA. The bending magnet beamline covers the energy range from 250 to $800 \mathrm{eV}$ with a photon flux of $10^{7}$ photon/s (Kilcoyne et al. 2003). Energy calibration was conducted by measuring the known spectral Rydberg line features in gaseous $\mathrm{CO}_{2}$ and $\mathrm{N}_{2}$ at their respective K-edges prior to the sample measurements. The absorption spectra (optical density, OD) were obtained as $\mathrm{OD}=-\ln \left(I / I_{0}\right)$, where $I$ is the $x$-ray intensity transmitted from the sample, and $I_{0}$ is the recorded without samples. The works of Leinweber et al. (2007) and Cody et al. (2008a) were used for absorption peak assignments.

\section{Results and discussion \\ Carbon-XANES}

Carbon elemental maps of RA-beforeTEM, RA-afterTEM, and $\mathrm{RB}$ reflect the homogenous carbon distributions across the entire particles (Figure 1). RA-beforeTEM and RB show similar C-K-edge-XANES spectra; peaks corresponding to aromatic/olefinic carbon $(\mathrm{C}=\mathrm{C})$ (peak $\mathrm{A}, 285.1 \mathrm{eV}$ ), aromatic ketone $(\mathrm{C}=\mathrm{C}-\mathrm{C}=\mathrm{O})$, nitrile $(\mathrm{C} \equiv \mathrm{N})$ and/or nitrogen heterocycles $(\mathrm{C}-\mathrm{N}=\mathrm{C}$ ) (peak $\mathrm{B}, 286.7 \mathrm{eV}$ ), and carboxyl carbon $(\mathrm{C}=\mathrm{O}(\mathrm{OH})$ ) (peak $\mathrm{C}, 288.2 \mathrm{eV}$ ) were observed (Figure 2). The spectra of the two regions of RB (R1 and R2 in Figure 1) were very similar, while the intensity of aromatic/olefinic $\mathrm{C}=\mathrm{C}$ peak $\mathrm{A}$ for $\mathrm{R} 1$ was higher than that for R2. The spectral intensities for RA-beforeTEM were much lower than those for RB. RA-afterTEM displayed peaks at similar energies in the C-XANES spectrum to those of the other two samples, although the overall absorption feature was rather broad (Figure 2). The developed peak B was shifted by $0.5 \mathrm{eV}$ from that of the other samples. This broad peak around $286.2 \mathrm{eV}$ could be a mixture of aromatic ketone $(C=C-C=O)$ and nitrile and/or nitrogen heterocycles. The peak $\mathrm{A}$ of aromatic/olefinic carbon was observed as a broad shoulder.

\section{Nitrogen-, oxygen-, and fluorine-XANES}

Nitrogen functional groups were identified from $R B$ and RA-afterTEM. In the N-K-edge-XANES spectrum of RB (Figure 3), imine $(\mathrm{C}=\mathrm{N}$ ) (peak $\mathrm{A}, 398.9 \mathrm{eV}$ ); nitrogen heterocycles $(\mathrm{C}-\mathrm{N}=\mathrm{C})$ and/or nitrile $(\mathrm{C} \equiv \mathrm{N})$ (peak $\mathrm{B}$, $399.7 \mathrm{eV}$ ); and an assembly of small peaks related to amide $\mathrm{N}$, pyrrolic $\mathrm{N}$, and amine $\mathrm{N}$ (peak $\mathrm{C}, 401$ to $402.5 \mathrm{eV}$ ) were identified. In the N-XANES spectrum of RA-afterTEM (Figure 3), peak B was shifted by $0.3 \mathrm{eV}$ from that of $R B$ and showed up only as a shoulder on the low-energy side of peak A.

Oxygen and F-K-edge XANES were continuously analyzed for RB (Figure 4). A peak at $531 \mathrm{eV}$ corresponded to the ketone group $(C=O)$. No features in the $x$-ray absorption were observed in the F-XANES range of 650 to $700 \mathrm{eV}$. 

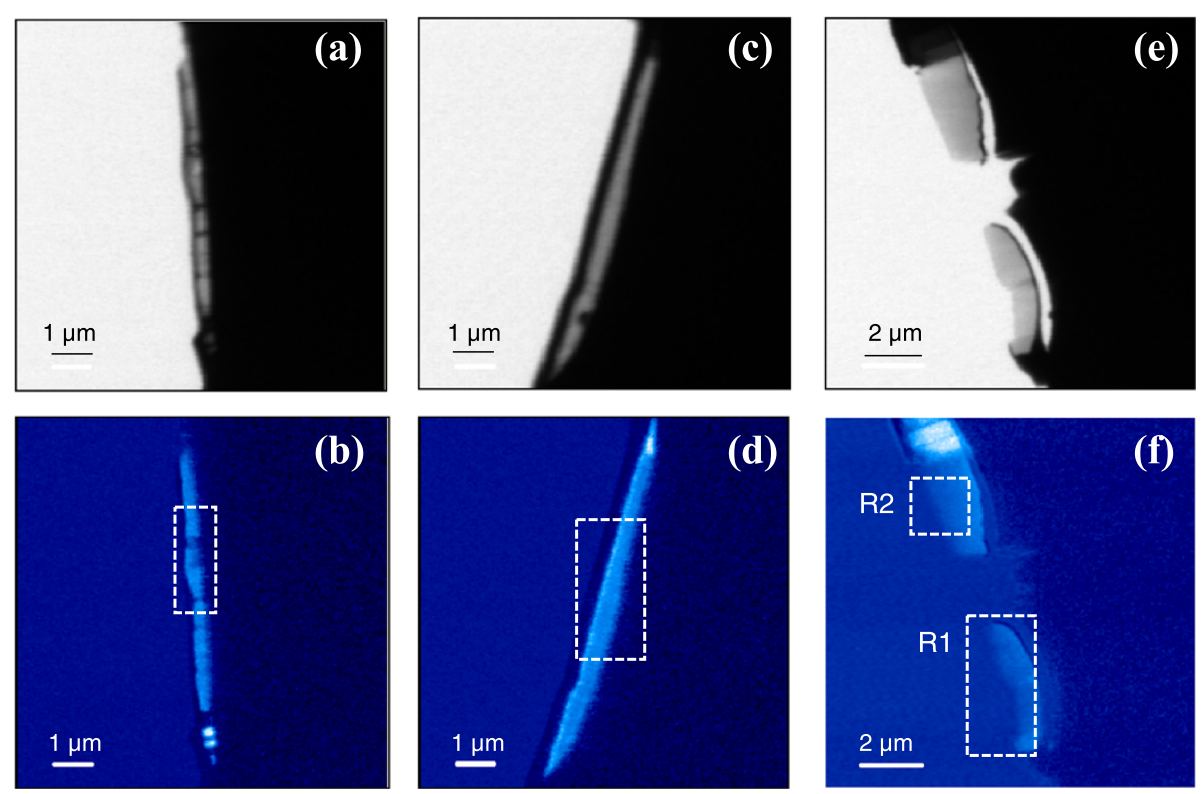

Figure 1 STXM images acquired at $350 \mathrm{eV}$ and carbon elemental maps. (a, b) RA-QD02-0120-beforeTEM (RA-beforeTEM), (c, d) RA-QD020120-afterTEM (RA- afterTEM), and (e, f) RB-QD04-0047-02 (RB). The dashed squares of the samples were analyzed to acquire the XANES spectra (in Figure 2).

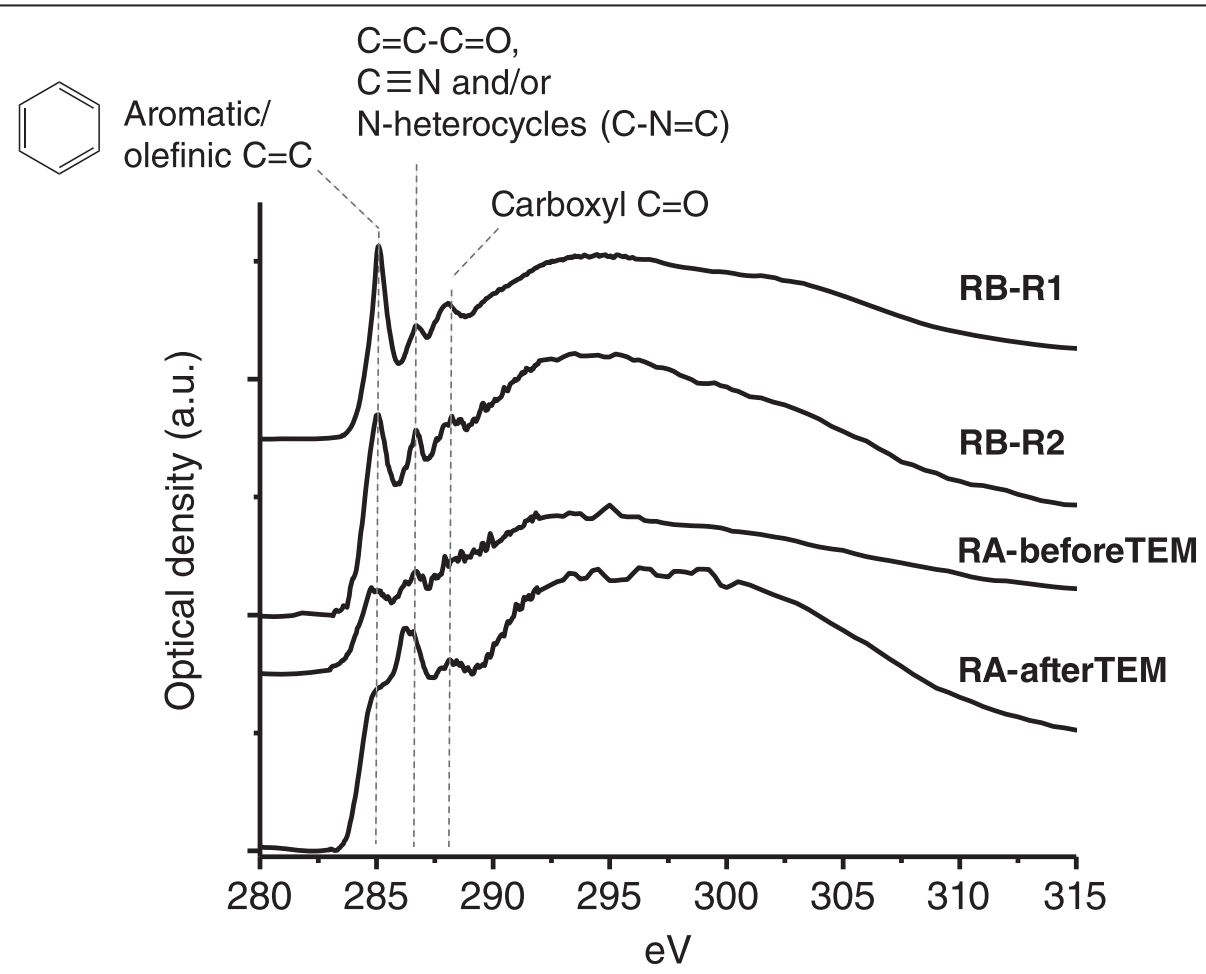

Figure 2 Carbon K edge-XANES spectra of RA-QD02-0120 and RB-QD04-0047-02. C-XANES spectra of RA-QD02-0120 before and after TEM observations (RA-before and after TEM) and two different regions (R1 and R2 in Figure 1) of RB-QD04-0047-02 (RB). The peaks assigned include the following: $1 \mathrm{~s}-\pi^{*}$ transition of aromatic/olefinic carbon $(C=C)$ at $285.1 \mathrm{eV} ; 1 \mathrm{~s}-\pi^{*}$ transition of vinyl-keto $(C=C-C=O)$, nitrile $(C \equiv N)$ and/or nitrogen heterocycles $(\mathrm{C}-\mathrm{N}=\mathrm{C})$ at $286.7 \mathrm{eV}$; and $1 \mathrm{~s}-\pi^{*}$ transition of carboxyl carbon $(\mathrm{COOH})$ at $288.2 \mathrm{eV}$. 


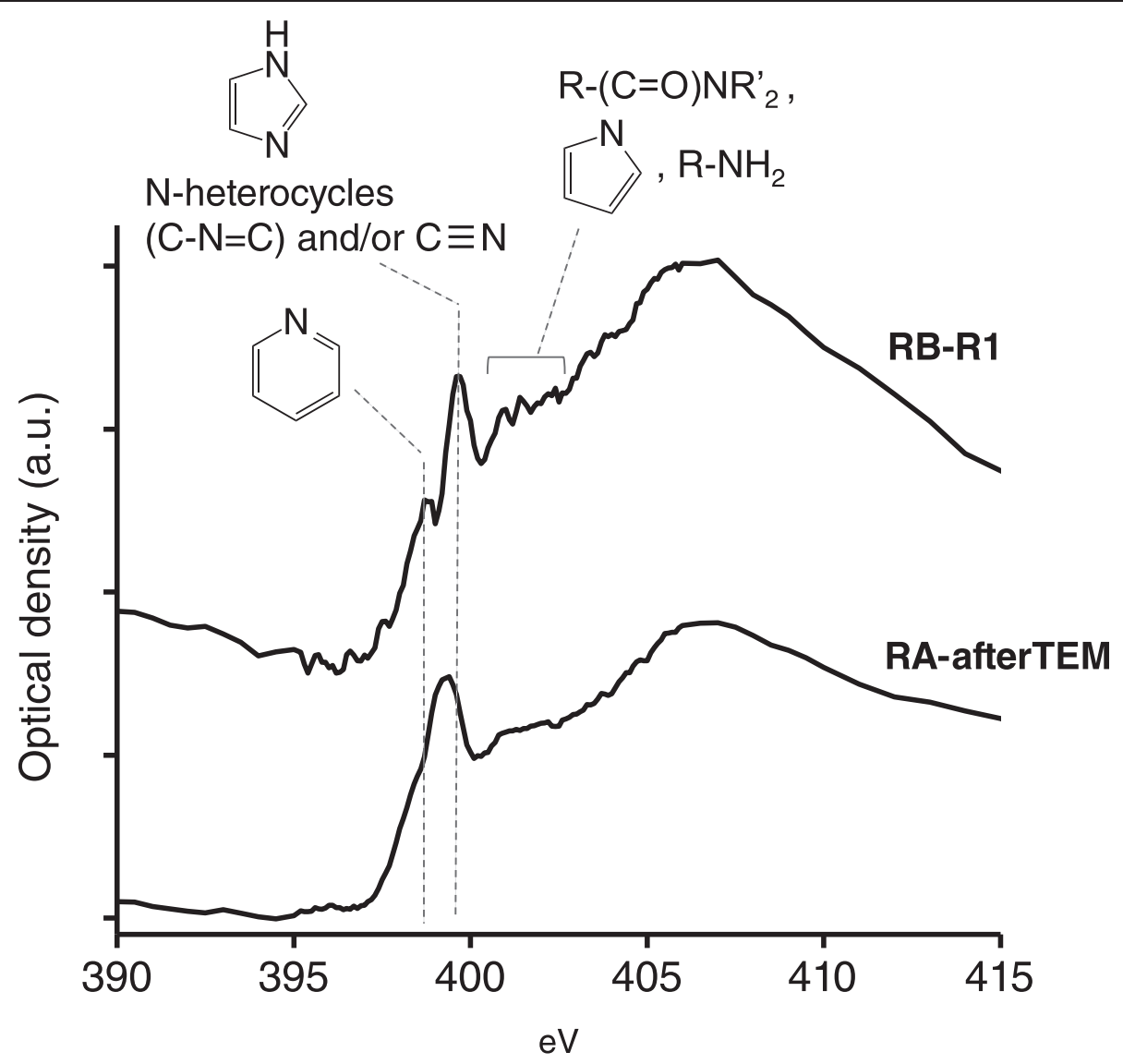

Figure 3 Nitrogen K edge-XANES spectra of RA-QD02-0120-afterTEM (RA-afterTEM) and RB-QD04-0047-02 (RB). The peaks assigned include the following: $1 \mathrm{~s}-\pi^{*}$ transition of imine $(C=N)$ at $398.9 \mathrm{eV} ; 1 \mathrm{~s}-\pi^{*}$ transition of nitrogen heterocycles $(C-N=C)$ and/or nitrile $(C \equiv N)$ at $399.7 \mathrm{eV}$; and an assembly of small peaks related to the $1 \mathrm{~s}-\pi^{*}$ transition of amide $\left(\mathrm{R}-(\mathrm{C}=\mathrm{O}) \mathrm{NR}_{2}^{\prime}\right)$ and the $1 \mathrm{~s}-3 \mathrm{p} / \mathrm{s}^{*}$ transitions of pyrrolic $\left(\mathrm{C}_{4} \mathrm{H}_{4} \mathrm{NH}\right)$ and amine $\left(\mathrm{R}-\mathrm{NH}_{2}\right)$ at 401 to $402.5 \mathrm{eV}$.

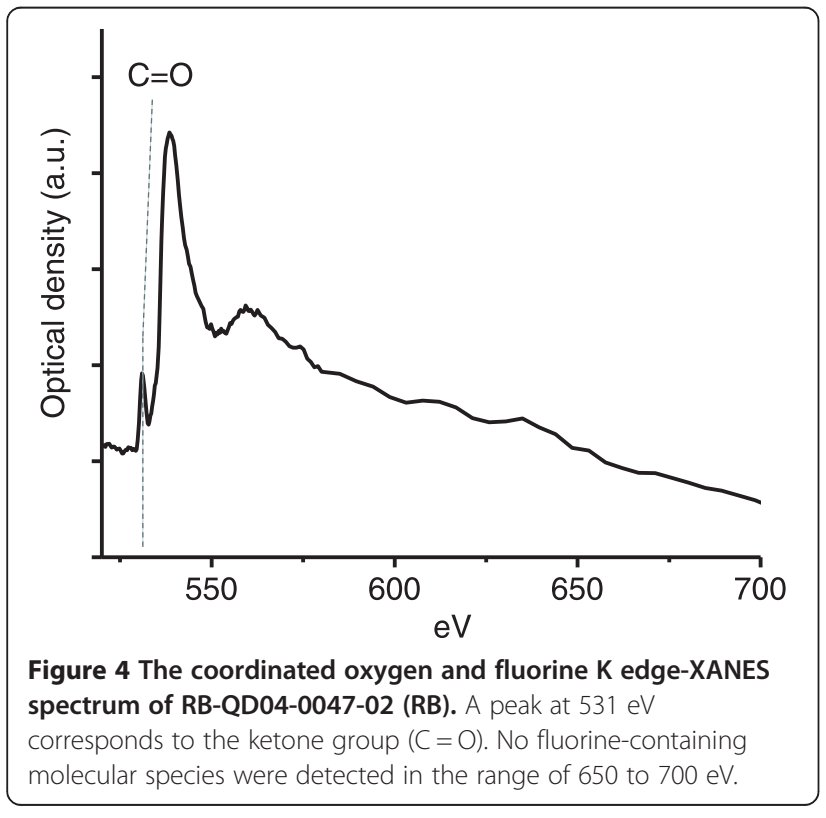

\section{Calcium-XANES}

A submicron-sized calcium grain was detected from the calcium elemental map of RA (Figure 5). The detection of calcium is compatible with the previous scanning transmission electron microscope (STEM) observations of calcium carbonate inclusions of approximately $300 \mathrm{~nm}$ in diameter that were taken from the same particle (Uesugi et al. 2014). The C-XANES spectrum of this spot exhibited a sharp peak at $290.5 \mathrm{eV}$ that was derived from carbonate. In the Ca- $\mathrm{L}_{2,3}$-edge-XANES spectrum of the same spot, peaks at $347.9,349.2,351.2$, and $352.5 \mathrm{eV}$ are observed positions that provide a good match to carbonates (Benzerara et al. 2004; Hanhan et al. 2009) (Figure 5). The spectral pattern was similar to that of a vaterite (Benzerara et al. 2004), although additional analyses will be necessary for more detailed characterization.

\section{Approach to identify category 3 carbonaceous particles}

Typical organic macromolecular features The two samples of category 3 carbonaceous particles, RA and RB, 
(a) C map

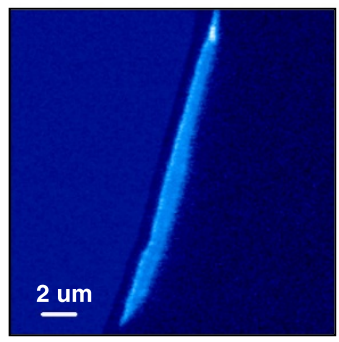

(b) Ca map

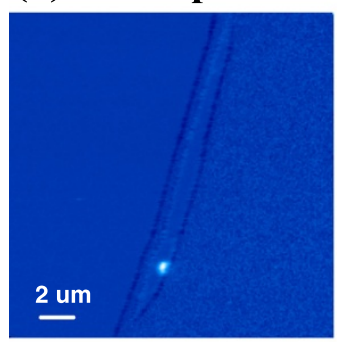

(c) Carbonate

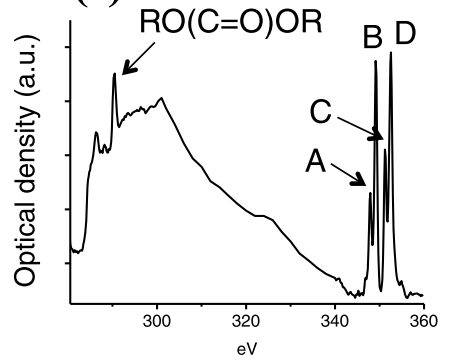

(d) B D

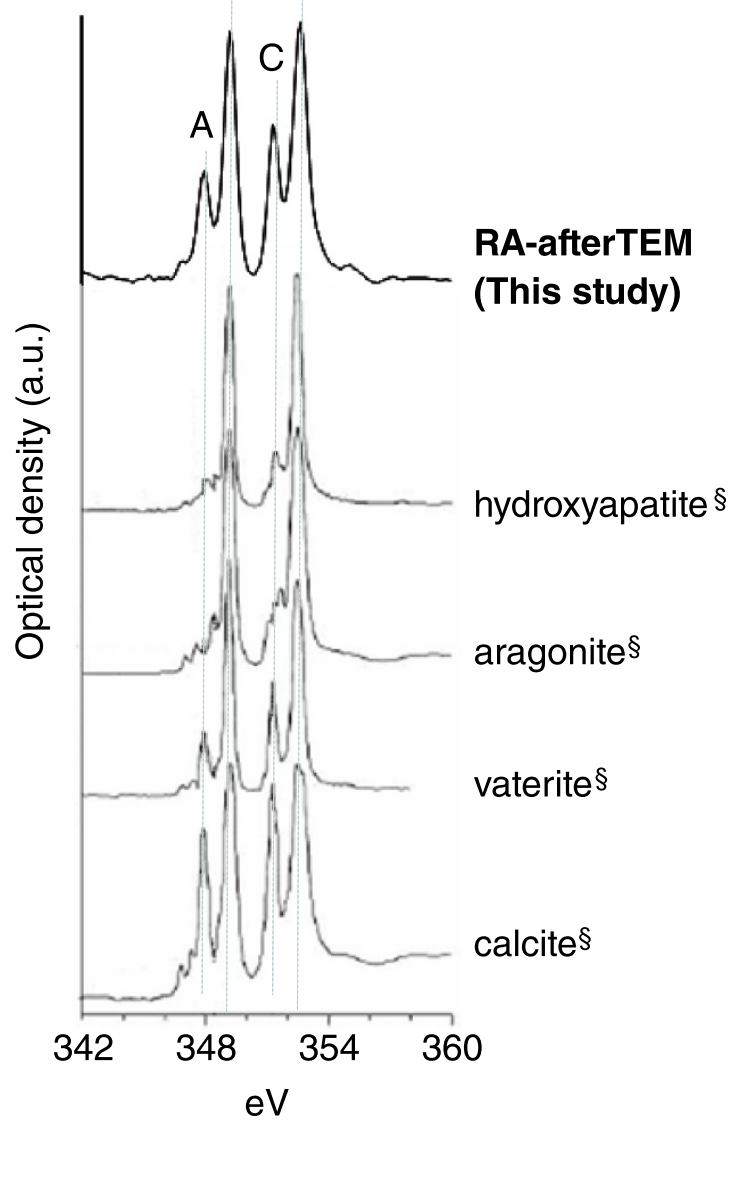

Figure 5 Elemental maps. Elemental maps of (a) carbon and (b) calcium for RA-QD02-0120-afterTEM (RA-afterTEM) that identify calcium spot within the carbonaceous particle. (c) The coordinated carbon $\mathrm{K}$ edge- and calcium $\mathrm{L}_{2,3}$ edge-XANES spectra of RA-afterTEM support the presence of calcium carbonate. A peak at $290.5 \mathrm{eV}$ is assigned to the $1 \mathrm{~s}-\pi^{*}$ transition of carbonate $\left(\mathrm{CO}_{3}\right)$. Two peaks around 349.2 (B) and $352.5 \mathrm{eV}(\mathrm{D})$ are assigned to the $L_{3}$ and $L_{2}$ edges of Ca, respectively. Two other smaller peaks ( $A$ and $C$ ) at 347.9 and $351.2 \mathrm{eV}$ are the coordination peaks of peaks A and B. (d) Comparison of nn enlarged spectrum of Ca-XANES for RA-afterTEM with those for hydroxyapatite, aragonite, vaterite, and calcite. ${ }^{\S}$ Ca-XANES spectra of carbonate standards (hydroxyapatite, aragonite, vaterite, and calcite) are derived from Benzerara et al. (2004).

displayed similar C-XANES spectra with regards to three coordination peaks. Their spectral patterns of aromatic/ olefinic carbon, aromatic ketone, and carboxyl carbon are typical of those observed from terrestrial coal (Cody et al. 1998; Bassim et al. 2012) and kerogen (Bernard et al. 2012) and even extraterrestrial organic solids from chondritic meteorites and IDPs (e.g., Flynn et al. 2003; Cody et al. 2011). This does not necessarily imply that our category 3 particles are coal or chondritic organics, but the findings do suggest the presence of some form of heterogeneous macromolecule formed through chemical processing in a natural environment. It is unlikely that the category 3 particles are derived from the LL 5 to 6 chondrite-like asteroid, since the C-XANES spectra show a lack of $1 \mathrm{~s}-\sigma^{*}$ excitons at $291.6 \mathrm{eV}$ corresponding to a highly conjugated $\mathrm{sp}^{2}$ carbon, which are generally characterized from thermally metamorphosed type 3+ chondrites (Cody et al. 2008b).

In contrast, the spectral pattern was distinct from those of the synthesized polymers (e.g., Ade et al. 1992; Urquhart et al. 1999) and biological polymers (e.g., Hitchcock et al. 2005), which are composed of specific molecular moieties. However, it is quite possible that the degradation of synthesized and biological polymers could convert their well-organized functional group structures into more heterogeneous macromolecules. Some of the synthetic polymer materials that were used on the Hayabusa spacecraft structures could potentially have been altered through chemical and physical processes (e.g., heat, cosmic ray irradiation) during the 7 years of Hayabusa's flight in deep space. If that is the case, the difference in the spectral intensities between 
RA (R1, R2) and RB might reflect different amounts of degradation of the same original source material. If this is the case, then the spectrum of RA-R1 may show the best vestiges of the original source material, since a sharp and high peak of aromatic carbon is a typical feature of various polymers (e.g., Ade et al. 1992; Urquhart et al. 1999).

Organic nitrogen macromolecules Typically, acidinsoluble organic solids from chondritic meteorites have less characteristic N-XANES spectra (Cody et al. 2008a) than those of the category 3 particles we examined here, despite the roughly similar C-XANES spectral patterns between the two materials. Rather, C- and N-XANES spectral patterns of the category 3 particles are somewhat similar to those seen in some of the organic materials found in Comet Wild 2 particles, in particular, organic nanoglobules (De Gregorio et al. 2010). However, most of the organic nanoglobules from extraterrestrial materials have isotopic anomalies in the form of deuterium and ${ }^{15} \mathrm{~N}$ enrichments (Nakamura-Messenger et al. 2006; De Gregorio et al. 2010), which represents a significant difference from our category 3 particles because they do not display isotopic anomalies (Ito et al. 2014).

The N-XANES spectral features of the category 3 particles enable the elimination of some materials used on the Hayabusa spacecraft. It is unlikely that the two category 3 particles are derived from Vectran and fluoro resin, which were used in the sampler horn of the Hayabusa spacecraft and in the clean chambers at the curation facility, respectively (Uesugi et al. 2014); this is because these polymers do not contain nitrogen. The lack of fluoropolymers is also supported by the absence of F-K-edge x-ray absorption at 650 to $700 \mathrm{eV}$ and characteristic peaks of the $\sigma^{*}(\mathrm{C}-\mathrm{F})$ resonance (Nagayama et al. 1995) in the C-XANES spectra.

In an ongoing investigation of the contamination coupons from the Hayabusa 2 spacecraft assembly clean room (unpublished data), nitrogen was detected by SEM-EDS and TEM analyses. However, the C-XANES spectra of the contamination coupons were distinct from those of the category 3 particles investigated in this study. This means that $\mathrm{N}$-containing contamination seems certain, but there may be multiple sources for its presence in the category 3 particles. Investigation of a variety of the $\mathrm{N}$-containing polymers used in and around the spacecraft, such as polyimide, will provide additional information needed for the future evaluation of spacecraft-related contamination.

Electron beam-sensitive material Different C-XANES spectral patterns of RA-beforeTEM and RA-afterTEM suggest that this category 3 particle has an electron beamsensitive composition. De Gregorio et al. (2010) compared the C-XANES spectra of an organic nanoglobule from a
Comet Wild 2 dust particle before and after TEM, and demonstrated that the original composition based on nitrile and carboxyl groups was converted to polyaromatic structures with a loss of carboxyls. The C-XANES spectrum of RA-afterTEM is quite similar to the 'post TEM' spectrum of the Comet Wild 2 organic nanoglobule. Such radiation-driven alterations may not be limited solely to extraterrestrial organic nanoglobules but might also occur for other polymer materials with similar molecular compositions. It was previously noted that the original composition of the organic nanoglobule is identical to cyanoacrylate (De Gregorio et al. 2010).

Similarly to TEM electron beam damage, ion beam damage from the FIB extraction of the samples is possible, although such damage is expected to be much smaller (Bassim et al. 2012). Nevertheless, identical FIB conditions were applied for all the samples in this study, so it is unlikely that spectral differences between the samples could be attributed to the FIB procedure alone.

Terrestrial or extraterrestrial? At this stage, it is premature to determine whether the category 3 carbonaceous particles we have examined are terrestrial or extraterrestrial. However, the general features of the particles are distinct from typical chondritic or IDP materials, in that they do not show any clear evidence of an extraterrestrial origin (e.g., isotopic anomalies and mineral compositions or textures). For instance, hydrogen isotopic compositions ( $\delta \mathrm{D})$ of insoluble organic matter from ordinary chondrites, which are meteorite groups similar to S-type asteroids, are approximately 2,000 to 5,000\% (Alexander et al. 2007). In contrast, the absence of high $\delta \mathrm{D}$ values from any particles of category 3 has lowered the likelihood of asteroidal origin (Ito et al. 2014). Nonetheless, considering that a number of isotopically normal organics have been reported in IDPs and micrometeorites (e.g., Messenger 2000; Yabuta et al. 2013), the extraterrestrial origin cannot be ruled out.

Clear differences in C-XANES spectra between a variety of polymers and the category 3 particles imply that the particles are not associated with fresh/unused synthesized materials or living organisms. However, there is a possibility that some type of nitrogen polymer material used in and around the spacecraft could have been degraded to form the materials seen in these particles. Degradation experiments of possible polymers, as well as the analysis of contamination coupons, will be necessary to further test this possibility. Although the origin of the calcium carbonate grain from RA-afterTEM is unknown, it will be important to check every possible source such as the carbonate filler that may have been used for polymer adhesives or the carbonate-based electrical double layer capacitors used in the Micro/Nano Experimental Robot Vehicle for Asteroid (MINERVA) that rode on the spacecraft. 


\section{Conclusions}

Two category 3 carbonaceous particles were analyzed by STXM using XANES. The molecular characteristics of the particles are summarized as follows:

1) The two carbonaceous particles have molecular features of aromatic/olefinic carbon, heterocyclic nitrogen and/or nitrile, and carboxyl carbon. The coordination of the carbon functional groups is consistent with a chemically heterogeneous organic macromolecule but distinct from industrial or biological 'fresh' polymers.

2) Various nitrogen functional groups including imine, nitrile, aromatic nitrogen, amide, amine, and pyrrole were identified from one of the particles, as well as ketone groups. In this regard, Vectran and fluororubber contaminants, which do not contain organic nitrogen, can be eliminated as the source of these category 3 particles.

3) The C-XANES spectra of the category 3 particles before and after TEM analysis were different, which demonstrates that the carbonaceous materials have electron beam-sensitive molecular structures.

4) No fluorine-containing molecular species were detected in the particles. This result indicates that 'fresh' fluoro-polymers can be excluded as the possible sources of these two category 3 samples.

5) Calcium-XANES spectrum and mapping has identified a calcium carbonate grain from one of the category 3 particles.

Although the origin of these two category 3 particles cannot be established at this time, it will be important to continue the chemical characterization of additional category 3 particles as well as model relevant material series and appropriate controls, including witness coupons. The knowledge obtained from these studies will help with the interpretation of samples obtained by Hayabusa 2, the next JAXA sample return mission. Hayabusa 2 is based on the Hayabusa configuration and aims to collect and characterize organic materials from the carbonaceous asteroid (162173) 1999 JU3.

\section{Competing interests}

The authors declare that they have no competing interests.

\section{Authors' contributions}

HY carried out the STXM analysis, XANES data interpretation, and manuscript preparation. MU carried out the immunoassays. MU, YK, TY, and YI carried out the sample handling, electron beam analyses, and interpretation. DK maintained a STXM instrument. HN, MI, SS, FK, HM, YT, TO, and MA participated in the design of the research and interpretation. All authors read and approved the final manuscript.

\section{Acknowledgements}

We appreciate Daniel Glavin, an anonymous reviewer, and official editor Michael Zolensky for their constructive comments, attentive corrections, and helpful editorial assistance. The STXM at the beam line 5.3.2.2 ALS facility is supported by the Director, Office of Science, Office of Basic Energy Sciences, and the US Department of Energy under Contract No. DE-AC02-05CH11231.

\section{Author details}

${ }^{1}$ Department of Earth and Space Science, Osaka University, 1-1 Machikaneyama, Toyonaka 560-0043 Osaka, Japan. ${ }^{2}$ Institute of Space and Astronautical Science (ISAS), Japan Aerospace Exploration Agency (JAXA), 3-1-1 Yoshinodai, Sagamihara 252-5210 Kanagawa, Japan. ${ }^{3}$ Department of Earth and Planetary Science, Faculty of Science, Kyushu University, Hakozaki, Fukuoka 812-8581, Japan. ${ }^{4}$ Kochi Institute for Core Sample Research, Japan Agency for Marine-Earth Science Technology (JAMSTEC), 200 Monobe Otsu, Nankoku 783-8502 Kochi, Japan. ${ }^{5}$ Advanced Light Source, 1 Cyclotron Rd, Berkeley 94720CA, USA. ${ }^{6}$ NASA Ames Research Center Moffett Field, Mountain View, CA 94035, USA. 'ife, Environment and Materials Science, Fukuoka Institute of Technology, 3-30-1 Wajiro-higashi, Fukuoka 811-0295, Japan. ${ }^{8}$ Department of Biogeochemistry, Japan Agency for Marine-Earth Science and Technology (JAMSTEC), 2-15 Natsushima, Yokosuka 237-0061, Japan.

Received: 30 April 2014 Accepted: 12 November 2014

Published online: 03 December 2014

\section{References}

Ade H, Zhang X, Cameron S, Costello C, Kirz J, Williams S (1992) Chemical contrast in $\mathrm{x}$-ray microscopy and spatially resolved XANES spectroscopy of organic solids. Science 258:972-975

Alexander CMO'D, Fogel M, Yabuta H, Cody GD (2007) The origin and evolution of chondrites recorded in the elemental and isotopic compositions of their macromolecular organic matter. Geochim Cosmochim Acta 71:4380-4403

Bassim ND, De Gregorio BT, Kilcoyne ALD, Scott K, Chou T, Wirick S, Cody GD, Stround RM (2012) Minimizing damage during FIB sample preparation of soft materials. J Microscopy 245:288-301

Benzerara K, Yoon TH, Tyliszczak T, Constantz B, Spormann AM, Brown GE Jr (2004) Scanning transmission X-ray microscopy study of microbial calcification. Geobiology 2:249-259

Bernard S, Horsfield B, Schulz HM, Wirth R, Schreiber A, Sherwood N (2012) Geochemical evolution of organic-rich shales with increasing maturity: a STXM and TEM study of the Posidonia shale (Lower Toarcian, northern Germany). Mar Petrol Geol 31:70-89

Busemann H, Nguyen AN, Cody GD, Hoppe P, Kilcoyne ALD, Stroud RM, Zega TJ, Nittler LR (2009) Ultra-primitive interplanetary dust particles from the comet 26P/Grigg-Skjellerup dust stream collection. Earth Planet Sci Lett 288:44-57

Cody GD, Ade H, Wirick S, Mitchell GD, Davis A (1998) Determination of chemical-structural changes in vitrinite accompanying luminescence alteration using C-NEXAFS analysis. Org Geochem 28:441-455

Cody GD, Ade H, Alexander CMO'D, Araki T, Butterworth A, Fleckenstein H, Flynn G, Gilles MK, Jacobsen C, Kilcoyne ALD, Messenger K, Sandford SA, Tyliszczak T, Westphal AJ, Wirick S, Yabuta H (2008a) Quantitative organic and lightelement analysis of comet 81P/Wild 2 particles using C-, N-, and O- XANES. Meteor Planet Sci 43:353-365

Cody GD, Alexander CMO'D, Yabuta H, Kilcoyne ALD, Araki T, Ade H, Dera P, Fogel M, Militzer B, Mysen BO (2008b) Organic thermometry for chondritic parent bodies. Earth Planet Sci Lett 272:446-455

Cody GD, Heying E, Alexander CMO'D, Nittler LR, Kilcoyne ALD, Sandford SA, Stroud RM (2011) Establishing a molecular relationship between chondritic and cometary organic solids. Proc Natl Acad Sci U S A 108:19171-19176

De Gregorio BT, Stroud RM, Nittler LR, Alexander CMO'D, Kilcoyne ALD, Zega TJ (2010) Isotopic anomalies in organic nanoglobules from comet 81P/wild 2: comparison to Murchison nanoglobules and isotopic anomalies induced in terrestrial organics by electron irradiation. Geochim Cosmochim Acta 74:4454-4470

Ebihara M, Sekimoto S, Shirai N, Hamajima Y, Yamamoto M, Kumagai K, Oura Y, Ireland TR, Kitajima F, Nagao K, Nakamura T, Naraoka H, Noguchi T, Okazaki R, Tsuchiyama A, Uesugi M, Yurimoto H, Zolensky ME, Abe M, Fujimura A, Mukai T, Yada T (2011) Neutron activation analysis of a particle returned from asteroid Itokawa. Science 333:1119-1121

Flynn GJ, Keller LP, Feser M, Wirick S, Jacobsen C (2003) The origin of organic matter in the solar system: evidence from the interplanetary dust particles. Geochim Cosmochim Acta 67:4791-4806

Flynn GJ, Wirick S, Keller LP (2013) Organic grain coatings in primitive interplanetary dust particles: implications for grain sticking in the solar nebula. Earth Planets Space 65:1159-1166 
Hanhan S, Smith AM, Obst M, Hitchcock AP (2009) Optimization of analysis of soft X-ray spectromicroscopy at the Ca 2p edge. J Electron Spectrosc Relat Phenom 173:44-49

Hitchcock AP, Morin C, Zhang X, Araki T, Dynes J, Stover H, Brash J, Lawrence JR, Leppard GG (2005) Soft X-ray spectromicroscopy of biological and synthetic polymer systems. J Electron Spectrosc Relat Phenom 144:259-269

Ito M, Uesugi M, Naraoka H, Yabuta H, Kitajima F, Mita H, Takano Y, Karouji Y, Yada T, Ishibashi Y, Okada T, Abe M (2014) H, C, and N isotopic compositions of Hayabusa category 3 organic samples. Earth Planet Space 66:91

Kilcoyne ALD, Tyliszczak T, Steele WF, Fakra S, Hitchcock P, Franck K, Anderson E, Harteneck B, Rightor EG, Mitchell GE, Hitchcock AP, Yang L, Warick T, Ade H (2003) Interferometer controlled scanning transmission microscopes at the Advanced Light Source. J Synchrotron Rad 10:125-136

Kitajima F, Kotsugi M, Ohkochi T, Naraoka H, Ishibashi Y, Abe M, Fujimura A, Okazaki R, Yada T, Nakamura T, Noguchi T, Nagao K, Tsuchiyama A, Mukai T, Sandford SA, Okada T, Shirai K, Ueno M, Yoshikawa M, Kawaguchi J (2011) A Micro-Spectroscopic Approach to the Carbonaceous Matter in the Particles Recovered by the Hayabusa Mission, (Abstract \#1855), 42nd Lunar and Planetary Science Conference

Kitajima F, Kotsugi M, Ohkochi T, Naraoka H, Ishibashi Y, Uesugi M, Karouji Y, Abe M, Fujimura A, Yada T, Okazaki R, Nakamura T, Noguchi T, Nagao K, Tsuchiyama A, Yurimoto H, Ebihara M, Ito M, Yabuta H, Mita H, Takano Y, Mukai T, Sandford SA, Okada T, Shirai K, Ueno M, Yoshikawa M, Kawaguchi J (2014) A micro-Raman and infrared spectrosopic approach to the several stony and organic (category 3) particles recovered by the Hayabusa mission. Earth Planet Space in press

Leinweber P, Kruse J, Walley FL, Gillespie A, Eckhardt K-U, Blyth RIR, Regier T (2007) Nitrogen K-edge XANES - an overview of reference compounds used to identify 'unknown' organic nitrogen in environmental samples. J Synchrotron Rad 14:500-511

Messenger S (2000) Identification of molecular-cloud material in interplanetary dust particles. Nature 404:968-971

Nagao K, Okazaki R, Nakamura T, Miura YN, Osawa T, Bajo K, Matsuda S, Ebihara M, Ireland TR, Kitajima F, Naraoka H, Noguchi T, Tsuchiyama A, Yurimoto H, Zolensky ME, Uesugi M, Shirai K, Abe M, Yada T, Ishibashi Y, Fujimura A, Mukai T, Ueno M, Okada T, Yoshikawa M, Kawaguchi J (2011) Irradiation history of Itokawa regolith material deduced from noble gases in the Hayabusa samples. Science 333:1128-1131

Nagayama K, Mitsumoto R, Araki T, Ouchi Y, Seki K (1995) Polarized XANES studies on the mechanical rubbing effect of poly(tetrafluoroethylene) and its model compound. Physica B 208\&209:419-420

Nakamura T, Noguchi T, Tanaka M, Zolensky ME, Kimura M, Tsuchiyama A, Nakato A, Ogami T, Ishida H, Uesugi M, Yada T, Shirai S, Fujimura A, Okazaki R, Sandford SA, Ishibashi Y, Abe M, Okada T, Ueno M, Mukai T, Yoshikawa M, Kawaguchi J (2011) Itokawa dust particles: a direct link between S-type asteroids and ordinary chondrites. Science 333:1113-1116

Nakamura-Messenger K, Messenger S, Keller LP, Clemett SJ, Zolensky ME (2006) Organic globules in the Tagish lake meteorite: remnants of the protosolar disk. Science 314:1439-1442

Naraoka H, Mita H, Hamase K, Mita M, Yabuta H, Saito K, Fukushima K, Kitajima F, Sandford SA, Nakamura T, Noguchi T, Okazaki R, Nagao K, Ebihara M, Yurimoto H, Tsuchiyama A, Abe M, Shirai K, Ueno M, Yada T, Ishibashi Y, Okada T, Fujimura A, Mukai T, Yoshikawa M, Kawaguchi J (2012) Preliminary organic compound analysis of microparticles returned from asteroid 25143 Itokawa by the Hayabusa mission. Geochem J 46:61-72

Naraoka H, Aoki D, Fukushima K, Uesugi M, Ito M, Kitajima F, Mita H, Yabuta H, Takano Y, Yada T, Ishibashi Y, Okada T, Abe M (2014) ToF-SIMS analysis of carbonaceous particles in the sample capsule of the Hayabusa mission. Earth Planet Space in press

Noguchi T, Nakamura T, Kimura M, Zolensky ME, Tanaka M, Hashimoto T, Konno M, Nakato A, Ogami T, Fujimura A, Abe M, Yada T, Mukai T, Ueno M, Okada T, Shirai K, Ishibashi Y, Okazaki R (2011) Incipient space weathering observed on the surface of Itokawa dust particles. Science 333:1121-1125

Sanford S, Aléon J, Alexander CMO'D, Araki T, Bajt S, Baratta GA, Borg J, Brucato JR, Burchell MJ, Busemann H, Butterworth A, Clemett SJ, Cody GD, Colangel L, Cooper G, D'Hendecourt L, Djouadi Z, Dworkin JP, Ferrini G, Fleckenstein H, Flynn GJ, Franchi IA, Fries M, Gilles MK, Glavin DP, Gounelle M, Grossemy F, Jacobsen C, Keller LP, Kilcoyne ALD et al (2006) Organics captured from comet wild 2 by the stardust spacecraft. Science 314:1720-1724

Tsuchiyama A, Uesugi M, Matsushima T, Michikami T, Kadono T, Nakamura T, Uesugi K, Nakano T, Sandford SA, Noguchi R, Matsumoto T, Matsuno J,
Nagano T, Imai Y, Takeuchi A, Suzuki Y, Ogami T, Katagiri J, Ebihara M, Ireland TR, Kitajima F, Nagao K, Naraoka H, Noguchi T, Okazaki R, Yurimoto H, Zolensky ME, Mukai T, Abe M, Yada T et al (2011) Three-dimensional structure of Hayabusa samples: origin and evolution of Itokawa regolith. Science 333:1121-1125

Uesugi M, Naraoka M, Ito M, Yabuta H, Kitajima F, Takano Y, Mita H, Ohnishi I, Kebukawa Y, Yada T, Karouji Y, Ishibashi Y, Okada T, Abe M (2014) Sequential analysis of carbonaceous materials in Hayabusa-returned samples for the determination of their origin. Earth Planet Space 66:102

Urquhart SG, Hitchcock AP, Smith AP, Ade HW, Lidy W, Rightor EG, Mitchell GE (1999) NEXAFS spectromicroscopy of polymers: overview and quantitative analysis of polyurethane polymers. J Electron Spectrosc Relat Phenom 100:119-135

Yabuta H, Noguchi T, Itoh S, Sakamoto N, Hashiguchi M, Abe K, Tsujimoto S, Kilcoyne ALD, Okubo A, Okazaki R, Tachibana S, Nakamura T, Terada K, Ebihara M, Nagahara H (2013) Evidence of Minimum Aqueous Alteration in Rock-Ice Body: Update of Organic Chemistry and Mineralogy of Ultracarbonaceous Antarctic Micrometeorite (Abstract \#2335), 44nd Lunar and Planetary Science Conference

Yada T, Fujimura A, Abe M, Nakamura T, Noguchi T, Okazaki R, Nagao K, Ishibashi Y, Shirai K, Zolenskt ME, Sandford S, Okada T, Uesugi M, Karouji Y, Ogawa M, Yakame S, Ueno M, Mukai T, Yoshikawa M, Kawaguchi J (2014) Hayabusareturned sample curation in the planetary material sample curation facility of JAXA. Meteorit Planet Sci 49:135-153

Yurimoto H, Abe K, Abe M, Ebihara M, Fujimura A, Hashiguchi M, Hashizume K, Ireland TR, Itoh S, Katayama J, Kato C, Kawaguchi J, Kawasaki N, Kitajima F, Kobayashi S, Meike T, Mukai T, Nagao K, Nakamura T, Naraoka H, Noguchi T, Okazaki R, Park C, Sakamoto N, Seto Y, Takei M, Tsuchiyama A, Uesugi M, Wakaki S, Yada T et al (2011) Oxygen isotopic compositions of asteroidal materials returned from Itokawa by the Hayabusa mission. Science 333:1116-1119

doi:10.1186/s40623-014-0156-0

Cite this article as: Yabuta et al:: X-ray absorption near edge structure spectroscopic study of Hayabusa category 3 carbonaceous particles. Earth, Planets and Space 2014 66:156.

\section{Submit your manuscript to a SpringerOpen ${ }^{\odot}$ journal and benefit from:}

- Convenient online submission

Rigorous peer review

- Immediate publication on acceptance

- Open access: articles freely available online

- High visibility within the field

- Retaining the copyright to your article

Submit your next manuscript at $>$ springeropen.com 